African Crop Science Journal by African Crop Science Society is licensed under a Creative Commons Attribution 3.0 Uganda License. Based on a work at www.ajol.info/ and www.bioline.org.br/cs DOI: http://dx.doi.org/10.4314/acsj.v24i3.1

\title{
IDENTIFICATION DE CARACTÉRISTIQUES AGRONOMIQUES POUR LE DIAGNOSTIC ET LA PRISE DE DÉCISION DE RÉGÉNÉRATION DES VERGERS DE CACAOYERS EN CÔTE D'IVOIRE
}

\author{
A.A. ASSIRI, O. DEHEUVELS ${ }^{1}$, Z.J. KELI' ${ }^{2}$, B.I. KEBE ${ }^{3}$, A. KONAN ${ }^{2}$ et N. KOFFI ${ }^{4}$ \\ Centre National de Recherche Agronomique (CNRA), BP 808 Divo, Côte d'Ivoire \\ ${ }^{1}$ Centre de coopération Internationale en Recherche Agronomique pour le Développement (CIRAD), TA 80/ \\ PS3 - Bd. de la Lironde, 34398 Montpellier Cedex 5, France \\ ${ }^{2}$ Centre National de Recherche Agronomique (CNRA), Direction Régionale de Man, BP 440 Man, Côte d'Ivoire \\ ${ }^{3}$ Centre National de Recherche Agronomique (CNRA), Direction Générale, 01 BP 1740 Abidjan 01, Côte d'Ivoire \\ ${ }^{4}$ Le Conseil du Café-Cacao, 04 BP 2576, Abidjan 04, Côte d'Ivoire \\ Auteur correspondant : alexis.assiri@yahoo.fr
}

(Receieved 19 February, 2016; accepted 8 June, 2016)

RÉSUMÉ

La régénération des vieux vergers de cacaoyers (Theobrama cacao L.) est une des stratégies mises en place en Côte d'Ivoire pour assurer une production durable de cacao. Une étude a été conduite en vue d'élaborer un guide pour réaliser un diagnostic rapide et adéquat des vergers et prendre la bonne décision de régénération, en optant judicieusement pour la réhabilitation ou la replantation. Un échantillon de 90 plantations de cacaoyers réhabilitées et de 75 autres replantées a été enquêté dans 12 départements représentatifs des trois principales zones de production de cacao du pays. Des données ont été collectées sur les caractéristiques agronomiques majeures de ces cacaoyères. Il s'est agi de la variété de cacaoyer, la superficie de la plantation, l'âge, le rendement, la densité de plantation, le nombre d'arbre d'ombrage permanent et le niveau des dégâts des insectes et des maladies. Les résultats ont montré que les critères discriminants des vergers régénérés sont l'âge, la densité de plantation et le

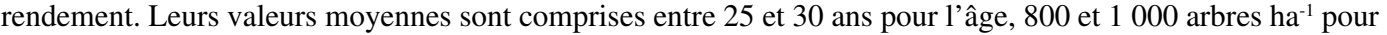
la densité et entre 250 et $400 \mathrm{~kg} \mathrm{ha}^{-1} \mathrm{an}^{-1}$ pour la production. A partir de ces trois critères et de leurs valeurs moyennes, un guide a été élaboré pour le diagnostic des vergers de cacaoyers et la prise de décision. Selon ce guide, les vieilles plantations de cacaoyers (plus de 25 ans), dégradées et improductives doivent être replantés. Cependant, les cacaoyères plus jeunes ayant des densités de plantation et des rendements plus élevés que les valeurs moyennes ci-dessus devraient être réhabilitées.

Mots Clés : Diagnostic, réhabilitation, replantation, Theobroma cacao

\begin{abstract}
The rehabilitation of the old cocoa (Theobrama cacao L.) farms is one of the major challenges for a sustainable cocoa production. A study was carried out to set up a guide which could be used as a decision making tool for a quick and efficient diagnosis of the old cocoa orchards and to choose the appropriate regeneration option (rehabilitation or replanting). A sample of 90 rehabilitated cocoa farms and of 75 replanted cocoa farms was surveyed in 12 regions representing the three main cocoa producing sectors in the country. Data were collected on the key agronomic characteristics of these cacao farms. These were cocoa variety, farm size, age, yield, planting density, number of shade trees and the level of damages caused by insects and diseases. The results showed that age, planting density and yield were the discriminating criteria of these farms. The average values of these criteria were 25 to 30 years for the age, 800 to 1000 trees ha ${ }^{-1}$ for the planting density and 250 to $400 \mathrm{~kg} \mathrm{ha}^{-1} \mathrm{an}^{-1}$ for the
\end{abstract}


yield. Based on these criteria and their average values, a decision making guide was designed for the diagnosis of cocoa farms and the choice of regeneration option. According to this guide, old cocoa farms (more than 25 years), degraded and unproductive should be replanted. However, younger farms having planting density and yield higher than the average values above should be rehabilitated.

Key Words: Diagnosis, rehabilitation, replanting, Theobroma cacao

\section{INTRODUCTION}

La culture du cacaoyer (Theobrama cacao L.) a été introduite en Côte d'Ivoire, dans la région est du pays, vers 1888 (Bardin, 1937 ; Burle, 1962). Elle a connu un développement rapide dans toute la moitié sud forestière du pays grâce aux atouts naturels dont le pays disposait et à la volonté politique des gouvernants (Assiri, 2010). Le principal atout naturel était la disponibilité d'importantes réserves forestières estimées à 16 millions d'hectares en 1960 (FAO, 2003). Les conditions écologiques, notamment la pluviométrie (plus de $1200 \mathrm{~mm} \mathrm{an}^{-1}$ ) et la majorité des sols étaient également favorables à la culture du cacaoyer (Perraud, 1971). Par ailleurs, la situation phytosanitaire était relativement bonne (Jouve et Milly, 1990 ; Losch, 1994 ; Petithuguenin, 1998).

Le développement de la cacaoculture s'est fait selon un mode de culture extensive et itinérante qui a entraîné le déplacement progressif des principaux secteurs de production de cacao de l'Est (avant 1960) vers le Centre-Ouest (1960 1980), puis le Sud-Ouest et l'Ouest du pays (à partir de 1980), suivant la dynamique des fronts pionniers (Losch, 1994 ; Ruf, 1997).

Grace à cet essor, le cacao est devenu, depuis plusieurs décennies, un produit agricole stratégique pour la Côte d'Ivoire, en raison de son rôle primordial dans la prospérité économique et sociale du pays. En effet, la Côte d'Ivoire en est le premier producteur mondial depuis 1978. Entre 2014 et 2015, la production ivoirienne a été de 1741000 tonnes, soit près de $42 \%$ de l'offre mondiale (ICCO, 2015). Au plan social, la culture du cacaoyer occupe une population agricole de plus d'un million de planteurs $(15 \%$ de la population rurale) et procure de nombreux emplois dans les secteurs secondaire et tertiaire (Anonyme, 1998 ; 2003). Au plan économique, le cacao procure environ $40 \%$ des recettes d'exportations de la Côte d'Ivoire et contribue, à hauteur de $15 \%$, au Produit Intérieur Brut (PIB) (Anonyme, 1999).

La durabilité de la production ivoirienne de cacao est cependant menacée par plusieurs contraintes, notamment le vieillissement des vergers et les difficultés de renouvellement de ceux-ci face à la pénurie de forêt, la baisse de la fertilité des sols et aux effets néfastes des perturbations climatiques. Le système traditionnel de culture extensive sur défriche forestière qui avait permis autrefois d'assurer le renouvellement du verger a atteint ses limites et ne peut plus être reproduit. La plupart des tentatives spontanées de régénération des vieux vergers se soldent par des échecs liés notamment à l'utilisation de techniques culturales peu adaptées.

Pour contribuer à assurer une production durable de cacao, des techniques appropriées de régénération cacaoyère ont été mises au point par la recherche (Assiri, 2010). Mais, certaines sont encore mal maîtrisées par les producteurs et les vulgarisateurs. Par ailleurs, ceux-ci ne disposent pas souvent d'outils permettant de réaliser un diagnostic adéquat des vergers et de prendre la bonne décision de régénération. Dans ce contexte, et face aux difficultés de régénération (Ruf, 2000 ; Ruf et Allangba, 2001), les producteurs abandonnent leurs vergers de cacaoyers dégradés ou les reconvertissent en plantations d'autres cultures pérennes, notamment le palmier à huile et l'hévéa (Naï Naï et al., 2000).

La présente étude a donc été entreprise en vue d'identifier les caractéristiques agronomiques les plus pertinentes qui peuvent être utilisées comme critères pour :

(i) effectuer un diagnostic rapide et adéquat des vieux vergers de cacaoyers ; 
(ii) prendre ensuite la bonne décision de régénération, en optant pour la réhabilitation ou la replantation.

\section{MATÉRIEL ET MÉTHODES}

Zone d'étude. Les enquêtes ont été réalisées dans les zones de production de cacao qui couvrent pratiquement la moitié sud de la Côte d'Ivoire (Fig. 1). Dans ces zones, le climat est de type subéquatorial, chaud et humide. Les hauteurs moyennes de pluie varient de 1100 à $1200 \mathrm{~mm}$ dans les régions centre à plus de $1500 \mathrm{~mm} \mathrm{an}^{-1}$ dans les régions sud. Les températures moyennes annuelles sont comprises entre 21 et $32^{\circ} \mathrm{C}$ (Kassin et al., 2008). La durée d'insolation (> 1700 heures

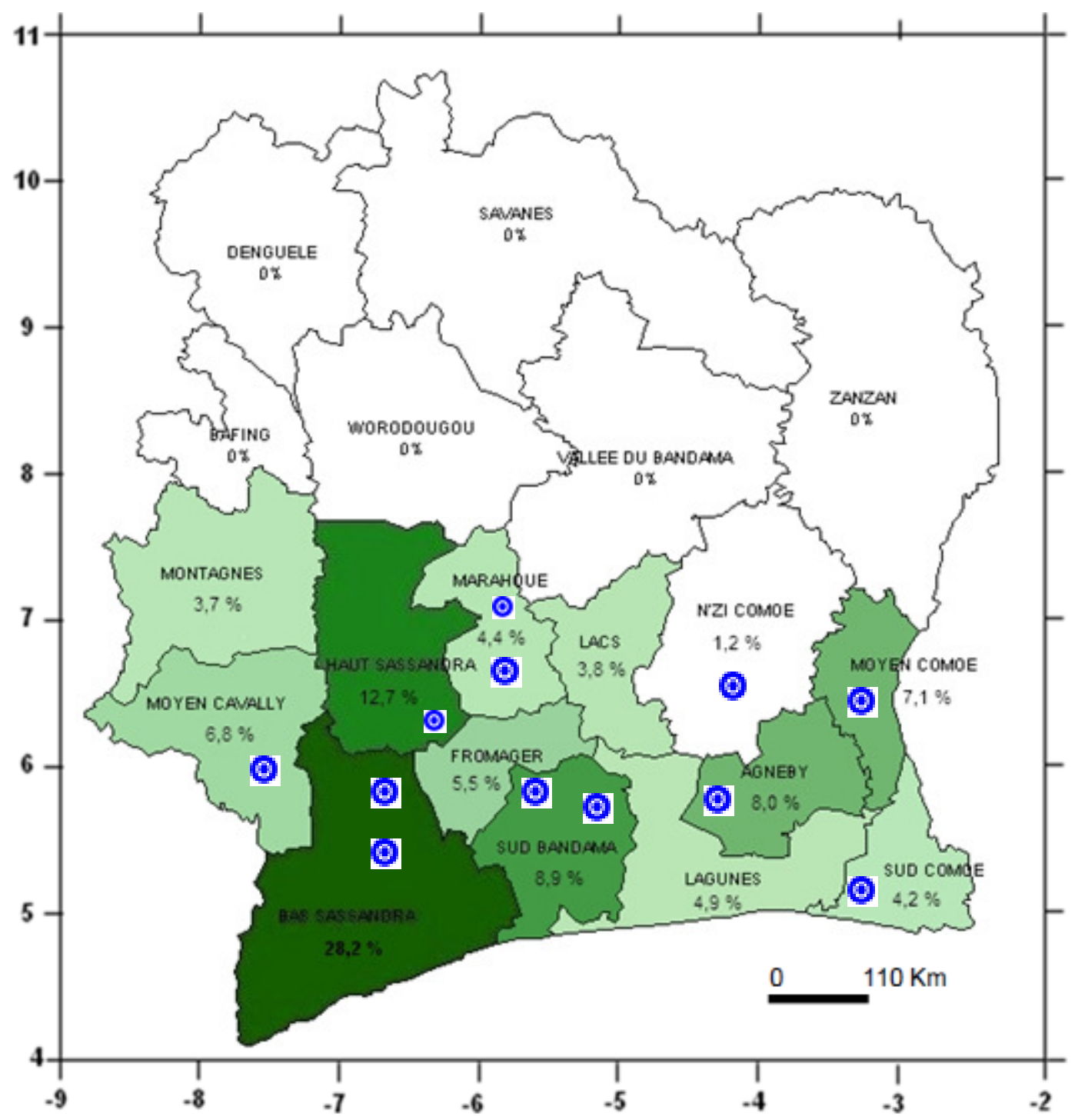

- Les chiffres sur la carte sont les pourcentages de superficie plantée par région

Figure 1. Sites des enquêtes en milieu paysan $(\mathbf{O}$ । dans les régions productrices de cacao. 
$\left.\mathrm{an}^{-1}\right)$ et l'humidité relative moyenne (>75\%) sont élevées durant toute l'année (Brou, 2005). La végétation naturelle était la forêt dense humide et couvrait près de 16 millions d'hectares dans les années 1960. Elle a été surexploitée et il n'en reste plus que des reliques estimées à moins de 2,5 millions d'hectares (FAO, 2003). Les sols sont en majorité des Ferralsols (FAO, 2009). Le pH est proche de 6,0 dans la couche $0-20 \mathrm{~cm}$ de profondeur. La fertilité chimique varie de faible à moyenne, avec des déficiences en phosphore et en potassium, au regard des exigences du cacaoyer (Assiri, 2010).

Méthode d'échantillonnage et choix des sites d'enquêtes. Une méthode d'échantillonnage stratifié a été employée pour sélectionner les sites d'enquêtes. Trois strates ont été considérées. Il s'est agi :

(i) d'abord, des trois principales zones de production de cacao qui sont l'Est, le CentreOuest et le Sud-Ouest ; elles représentent dans le temps et l'espace, les grandes vagues d'extension des vergers de cacaoyers en Côte d'Ivoire (Losch, 1994 ; Ruf, 1995) ;

(ii) ensuite, les départements dans chaque zone de production ; 12 départements de forte production de cacao ont été choisis sur la base des résultats du recensement national de l'agriculture en 2001 ;

(iii) enfin, les villages; un total de 30 villages ont été identifiés à l'issue de visites de terrain afin de bien couvrir les départements retenus.

Choix des producteurs et des parcelles de cacaoyers. Les enquêtes ont porté sur les cacaoyères replantées ou réhabilitées dans chaque village. Pour sélectionner effectivement ces parcelles, l'approche suivante a été suivie :

(i) des réunions de sensibilisation des producteurs et d'explication des notions de réhabilitation et de replantation cacaoyère ont été tenues dans les villages et les campements rattachés ; (ii) à l'issue de ces réunions, des listes provisoires des producteurs, donc des parcelles de cacaoyers replantées ou réhabilitées ont été établies ;

(iii) des visites de confirmation des parcelles sur le terrain ont été ensuite effectuées ; elles ont permis d'établir les listes définitives par village des parcelles de cacaoyers répondant effectivement aux critères de réhabilitation ou de replantation; ainsi, pour la réhabilitation, 90 plantations couvrant 234,5 ha ont été enquêtées dans 27 villages. Dans le cas de la replantation, 75 parcelles de 70,75 ha au total ont été enquêtées dans 28 villages (Tableau 1).

Réalisation des enquêtes et données collectées. Les enquêtes ont été effectuées par sept enquêteurs pendant huit mois dont 4,5 mois pour la replantation et 3,5 mois pour la réhabilitation. Deux méthodes (quantitative et qualitative) de collecte d'informations ont été utilisées.

La méthode qualitative a consisté en des interviews en utilisant un questionnaire d'enquête pour la replantation d'une part, et un autre pour la réhabilitation d'autre part. Les interviews étaient individuelles et ont eu lieu dans les parcelles de cacaoyers. Les données collectées ont concerné les caractéristiques agronomiques des cacaoyères avant d'être soumises à la replantation ou à la réhabilitation. Il s'est agi essentiellement de la variété de cacaoyer cultivée, le dispositif de plantation, la superficie, l'âge de la plantation et le rendement.

La méthode quantitative a consisté en des observations directes dans les plantations enquêtées. A l'aide de formulaire-guides joints aux fiches d'enquête, cette méthode a permis :

(i) d'estimer la densité de plantation par la méthode des carrés. Au moins trois carrés de $100 \mathrm{~m}^{2}(10 \mathrm{~m} \times 10 \mathrm{~m})$ ont été délimités dans chaque plantation. Le nombre moyen de cacaoyers par carré a été multiplié par 100 pour obtenir la densité à l'hectare ; 
TABLEAU 1. Nombre de parcelles de cacaoyers enquêtées par type de régénération en fonction des zones de production et des départements

\begin{tabular}{|c|c|c|c|c|c|}
\hline \multirow[t]{2}{*}{ Zones de production de cacao } & \multirow[t]{2}{*}{ Départements } & \multicolumn{2}{|c|}{ Réhabilitation } & \multicolumn{2}{|c|}{ Replantation } \\
\hline & & $\begin{array}{l}\text { Nombre de } \\
\text { plantations }\end{array}$ & $\begin{array}{l}\text { Superficie } \\
\text { (ha) }\end{array}$ & $\begin{array}{c}\text { Nombre de } \\
\text { parcelles }\end{array}$ & $\begin{array}{c}\text { Superficie } \\
\text { (ha) }\end{array}$ \\
\hline \multirow[t]{4}{*}{ Est et Sud-Est } & Abengourou & 14 & 54,5 & 17 & 31,5 \\
\hline & Aboisso & 2 & 2,25 & 18 & 16,75 \\
\hline & Agboville & 12 & 33,0 & - & - \\
\hline & Bongouanou & 13 & 18,0 & - & - \\
\hline \multirow[t]{4}{*}{ Centre-Ouest } & Bouaflé & 7 & 15,0 & 7 & 8,5 \\
\hline & Divo, Lakota & 23 & 83,0 & 9 & 3,0 \\
\hline & Issia & 5 & 5,0 & - & - \\
\hline & Sinfra & 3 & 2,0 & - & - \\
\hline \multirow[t]{2}{*}{ Ouest et Sud-Ouest } & Guiglo & 8 & 10,75 & 10 & 3,5 \\
\hline & Soubré, Méagui & 3 & 11,0 & 14 & 7,5 \\
\hline Total & 12 & 90 & 234,5 & 75 & 70,75 \\
\hline
\end{tabular}

(ii) de déterminer le mode de conduite des plantations en relation avec la présence ou l'absence d'arbres d'ombrage permanent dans les cacaoyères. Il a été apprécié en évaluant l'ombrage permanent selon l'échelle de Freud et al. (2000) :

- plein soleil : pas d'arbres d'ombrage, mais présence éventuelle d'arbres fruitiers ;

- ombrage faible à léger : moins de 10 grands arbres forestiers par hectare ;

- ombrage moyen à fort : plus de 10 grands arbres forestiers par hectare.

(iii) d'apprécier l'état sanitaire et végétatif des cacaoyers selon trois niveaux (aucun, faible, élevé) des dégâts provoqués par les insectes et les maladies.

Les données quantitatives et qualitatives collectées ont été saisies sur le tableur EXCEL. Les analyses statistiques (statistiques descriptives, analyses de variances, comparaison multiples des moyennes) ont été faites avec le logiciel XLSTAT.

\section{RÉSULTATS}

\section{Caractéristiques agronomiques des cacaoyères avant la replantation}

Âge des plantations. L'âge des cacaoyères replantées a varié de 11 à 70 ans. La répartition de ces vergers $(n=75)$ par classe d'âges a révélé que (Tableau 2) les cacaoyères fréquemment remplacées sont celles de :

(i) plus de 40 ans $(64,9$ à 75,1 \%) à l'Est et au Sud-Est ;

(ii) de 30 à 50 ans $(81,0$ à 93,8\%) au Centre-Ouest ;

(iii) de 10 à 30 ans (90,8 à $100 \%$ ) à l'Ouest et au Sud-Ouest.

Ainsi, les moyennes d'âge des vergers replantés étaient comprises entre 25,6 et 43,0 ans. Elles sont significativement différentes $(\mathrm{P}<0,01 \%)$ d'une zone de production à une autre (Tableau 3 ).

Densité de plantation et rendement. Avant la replantation, les vieux vergers de cacaoyers avaient des densités moyennes inférieures à 1000 pieds ha ${ }^{-1}$. Ils produisaient en moyenne moins de 
TABLEAU 2. Répartition des superficies replantées (en \%) par département en fonction de l'âge des précédents cacaoyers

\begin{tabular}{|c|c|c|c|c|c|c|}
\hline \multirow[t]{2}{*}{ Classes d'âges } & \multicolumn{2}{|c|}{ Est et Sud-Est } & \multicolumn{2}{|c|}{ Centre-Ouest } & \multicolumn{2}{|c|}{ Sud-Ouest et Ouest } \\
\hline & Abengourou & Aboisso & Divo & Bouaflé & Guiglo & Soubré \\
\hline$<10$ ans & - & - & - & - & - & - \\
\hline $11-20$ & 4,4 & 4,0 & - & - & 59,1 & 35,6 \\
\hline $21-30$ & 13,1 & 4,8 & 6,2 & 19 & 40,9 & 55,2 \\
\hline $31-40$ & 17,6 & 16,1 & 68,8 & 28,6 & - & 9,2 \\
\hline $41-50$ & 47,3 & 59,8 & 25,0 & 52,4 & - & - \\
\hline$>50$ & 17,6 & 15,3 & - & 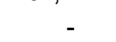 & - & - \\
\hline
\end{tabular}

- : aucune plantation appartenantà la classe d'âges

TABLEAU3. Moyennes d'âge, densités et rendements des vieux vergers de cacaoyers remplacés par de nouvelles cacaoyères en fonction des zones de production

\begin{tabular}{lcrr}
\hline Zones de production & $\begin{array}{c}\text { Âge moyen (années) des } \\
\text { cacaoyères replantées }\end{array}$ & $\begin{array}{r}\text { Densité moyenne } \\
\left(\text { cacaoyers ha }{ }^{-1}\right)\end{array}$ & $\begin{array}{c}\text { Rendement } \\
\left(\mathrm{kg} \mathrm{ha}^{-1} \mathrm{an}^{-1}\right)\end{array}$ \\
\hline Est et Sud-Est & $43,0 \pm 6,3 \mathrm{a}$ & 100 & $145,7-329,7$ \\
Centre-Ouest & $32,6 \pm 7,7 \mathrm{ab}$ & 250 & $120,0-155,1$ \\
Sud-Ouest et Ouest & $25,6 \pm 4,6 \mathrm{~b}$ & $300-1000$ & $137,7-160,0$ \\
Signification des différences entre les moyennes & $* * *$ & - & \\
\hline
\end{tabular}

*** : Différence significative à $\mathrm{P}<0,01 \% ; a, b, c$ : Les moyennes suivies de la même lettre ne sont pas statistiquement différentes (Test de Bonferroni, $\mathrm{P}<5 \%$ )

$329,7 \mathrm{~kg}$ de cacao par hectare (Tableau 3). Certains vergers remplacés n'étaient plus entretenus ni exploités. On n’y dénombrait que quelques pieds de cacaoyers disséminés dans les plantations.

\section{Caractéristiques agronomiques des cacaoyères soumises à la réhabilitation}

Taille et matériel végétal planté. Les superficies des plantations réhabilitées ont varié de 0,125 à 16 ha. Les plantations couvrant moins de 3 ha représentaient 75,9\% des cacaoyères enquêtées (Fig. 2). Trois variétés de cacaoyers ont été plantées. Les variétés non sélectionnées (76,7 \% des plantations enquêtées) et la variété Amelonado (45,6 \%) ont été les plus cultivées (Tableau 4). Dans $91 \%$ des plantations, les cacaoyers n'ont pas été plantés en ligne.
Âge des cacaoyères réhabilitées. L'âge des cacaoyères réhabilitées a varié de 6 à 64 ans. La majorité $(64,3 \%)$ avait un âge compris entre 10 et 30 ans (Fig. 3). Les moyennes d'âges ont été significativement différentes $(\mathrm{P}<0,0001)$ d'un département à un autre (Tableau 5). On a distingué :

(i) les vergers de 15 à 25 ans, à Aboisso, Guiglo, Issia, Agboville, Sinfra et Divo ;

(ii) les plantations de 25 à 35 ans, à Abengourou, Bouaflé et Soubré ;

(iii) les cacaoyères de plus de 35 ans dans le département de Bongouanou. 


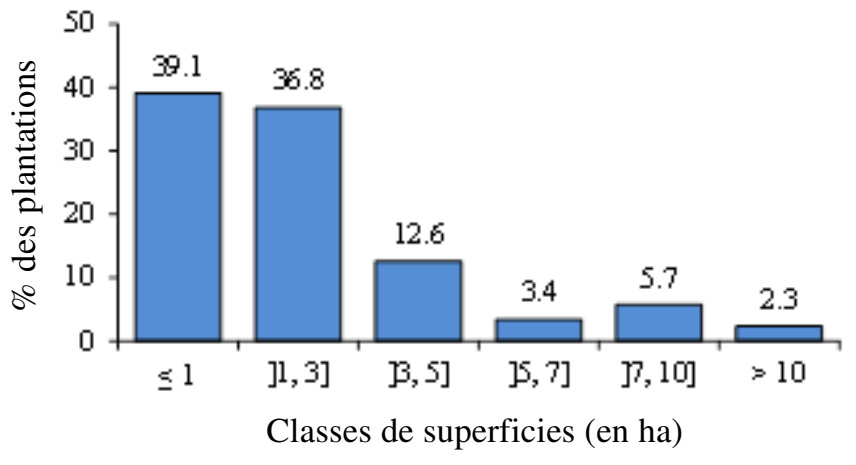

Figure 2. Répartition des cacaoyères réhabilitées en fonction de leurs superficies $(n=90)$.

TABLEAU 4. Répartition des vergers de cacaoyers réhabilités en fonction du matériel végétal planté et du dispositif de plantation $(n=90)$

\begin{tabular}{lcccccc}
\hline & \multicolumn{2}{c}{ Matériel végétal planté } & & & \multicolumn{2}{c}{ Dispositif de plantation } \\
\cline { 2 - 3 } \cline { 5 - 7 } & $\begin{array}{c}\text { Variétés non } \\
\text { sélectionnées }\end{array}$ & $\begin{array}{c}\text { Amelonado } \\
\text { (cacao français) }\end{array}$ & Hybride & & Non aligné & En ligne \\
\hline \% des vergers enquêtés & 76,7 & 45,6 & 8,9 & & 91,0 & 9,0 \\
\hline
\end{tabular}

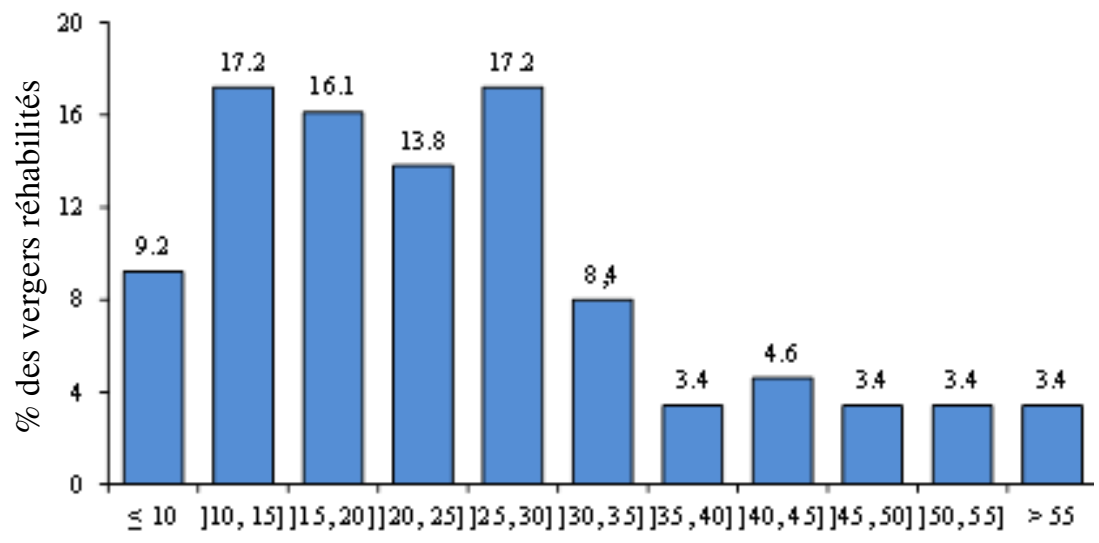

Classes d'âges des vergers (en années)

Figure 3. Répartition des plantations réhabilitées (en \%) par classe d'âges $(n=90)$.

Mode de conduite, densités et rendements des plantations réhabilitées. Les cacaoyères réhabilitées étaient conduites selon trois modes : le plein soleil, l'ombrage permanent léger et l'ombrage permanent dense (Tableau 6). La conduite en plein soleil a caractérisé les vergers réhabilités au Sud-Ouest, à l'Ouest et au Centre-
Ouest (55,3 à 63,6\% des plantations). Par contre, à l'Est et au Sud-Est, la majorité des plantations réhabilitées (39,0 à 48,8\%) était conduite sous un ombrage permanent.

Une diminution de la densité et des rendements moyens a été observé avec l'âge des vergers. Dans les quatre classes d'âges (10 à 30 
TABLEAU 5. Âges moyens des plantations de cacaoyers réhabilitées dans les différents départements enquêtés (nombre de parcelles $=90$ )

\begin{tabular}{lc}
\hline Département & Âge moyen des plantations (années) \\
\hline Bongouanou & $41,8 \pm 9,3 \mathrm{a}$ \\
Soubré & $34,5 \pm 13,2 \mathrm{ab}$ \\
Bouaflé & $32,3 \pm 8,2 \mathrm{ab}$ \\
Abengourou & $26,1 \pm 8,5 \mathrm{~b}$ \\
Divo & $25,1 \pm 4,0 \mathrm{bc}$ \\
Sinfra & $22,5 \pm 3,5 \mathrm{bc}$ \\
Agboville & $18,3 \pm 3,5 \mathrm{bc}$ \\
Issia & $17,8 \pm 6,6 \mathrm{bc}$ \\
Guiglo & $15,4 \pm 2,8 \mathrm{c}$ \\
Aboisso & $15,0 \pm 1,4 \mathrm{c}$ \\
\hline
\end{tabular}

a, b, c : Les moyennes suivies de la même lettre ne sont pas statistiquement différentes (Test de Bonferroni, $P<5 \%$ ) ans) les plus représentées (cf. Fig. 3), les densités moyennes des plantations ont varié de 1250 à 1450 pieds de cacaoyers ha-1, et les rendements moyens, de 250 à $380 \mathrm{~kg} \mathrm{ha}^{-1} \mathrm{an}^{-1}$ (Fig. 4). À partir de 35 ans, les densités étaient comprises entre 800 et 1000 arbres $\mathrm{ha}^{-1}$ et les rendements ont oscillé autour de $150 \mathrm{~kg} \mathrm{ha}^{-1} \mathrm{an}^{-1}$.

Caractéristiques agronomiques comparées des vergers régénérés. Les cacaoyères replantées $(\mathrm{n}=75)$ avaient un âge moyen de 38,4 ans, contre 25,9 ans pour les plantations réhabilitées (Tableau 7). Ils avaient des densités (179,0 pieds ha-1) et des rendements moyens $\left(191,2 \mathrm{~kg} \mathrm{ha}^{-1}\right)$ très faibles, par rapport à ceux des cacaoyères réhabilitées (1 237,0 pieds ha $^{-1}$ pour $331,0 \mathrm{~kg}$ $\left.\mathrm{ha}^{-1}\right)$. Les analyses de variance ont montré que

TABLEAU 6. Modes de conduite (\% des plantations) des vergers réhabilités dans les secteurs de production de cacao (nombre de parcelles $=90$ )

\begin{tabular}{lccc}
\hline Secteurs de production de cacao & \multicolumn{3}{c}{ Modes de conduite des vergers de cacaoyers } \\
\cline { 2 - 4 } & Plein soleil & Ombrage faible à léger & Ombrage moyen à fort \\
\hline Est, Sud-Est & 12,2 & 48,8 & 39,0 \\
Centre-Ouest & 55,3 & 23,7 & 21,0 \\
Ouest, Sud-Ouest & 63,6 & 9,1 & 27,3 \\
\% pondéré & 36,7 & 33,3 & 30,0 \\
\hline
\end{tabular}

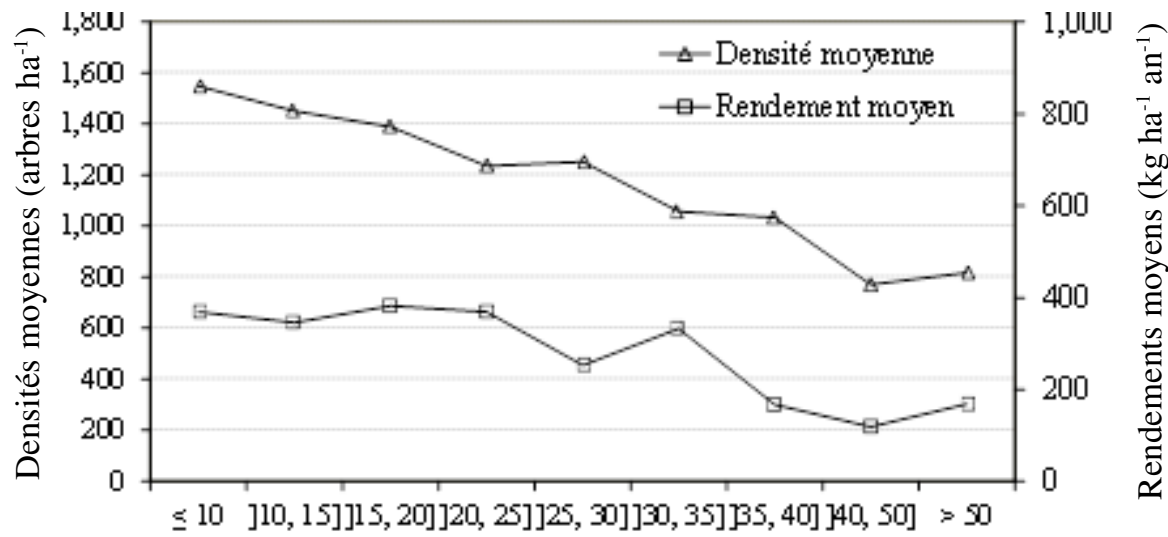

Classes d'âges des vergers

Figure 4. Densités moyennes de plantation et rendements moyens des vergers de cacaoyers par classe d'âges avant la réhabilitation $(n=90)$. 
TABLEAU 7. Caractéristiques initiales comparées des vergers de cacaoyers régénérés

\begin{tabular}{lrrr}
\hline Paramètres & \multicolumn{1}{c}{$\begin{array}{c}\text { Cacaoyères } \\
\text { réhabilitées }(\mathrm{n}=90)\end{array}$} & $\begin{array}{c}\text { Cacaoyères } \\
\text { replantées }(\mathrm{n}=75)\end{array}$ & $\begin{array}{c}\text { Différence entre } \\
\text { les moyennes }\end{array}$ \\
\hline Âge moyen des vergers (années) & $25,9 \pm 2,9$ & $38,4 \pm 5,0$ & $* * *$ \\
Densité moyenne de plantation (nombre d'arbres ha-1) & $1237,0 \pm 113,7$ & $179,0 \pm 75,7$ & $* * *$ \\
Rendement moyen $\left(\mathrm{kg} \mathrm{ha}^{-1}\right)$ & $331,0 \pm 47,7$ & $191,2 \pm 53,5$ & $* * *$ \\
\hline
\end{tabular}

${ }^{* * *}$ : différence entre moyennes significative à $P<0,01 \% . n$ : nombre de parcelles de cacaoyers enquêtées

Critère 1 :

$\hat{A}$ ge du verger

Critère 2 :

Densité de plantation

\section{Critère 3 :}

Rendem ent moyen

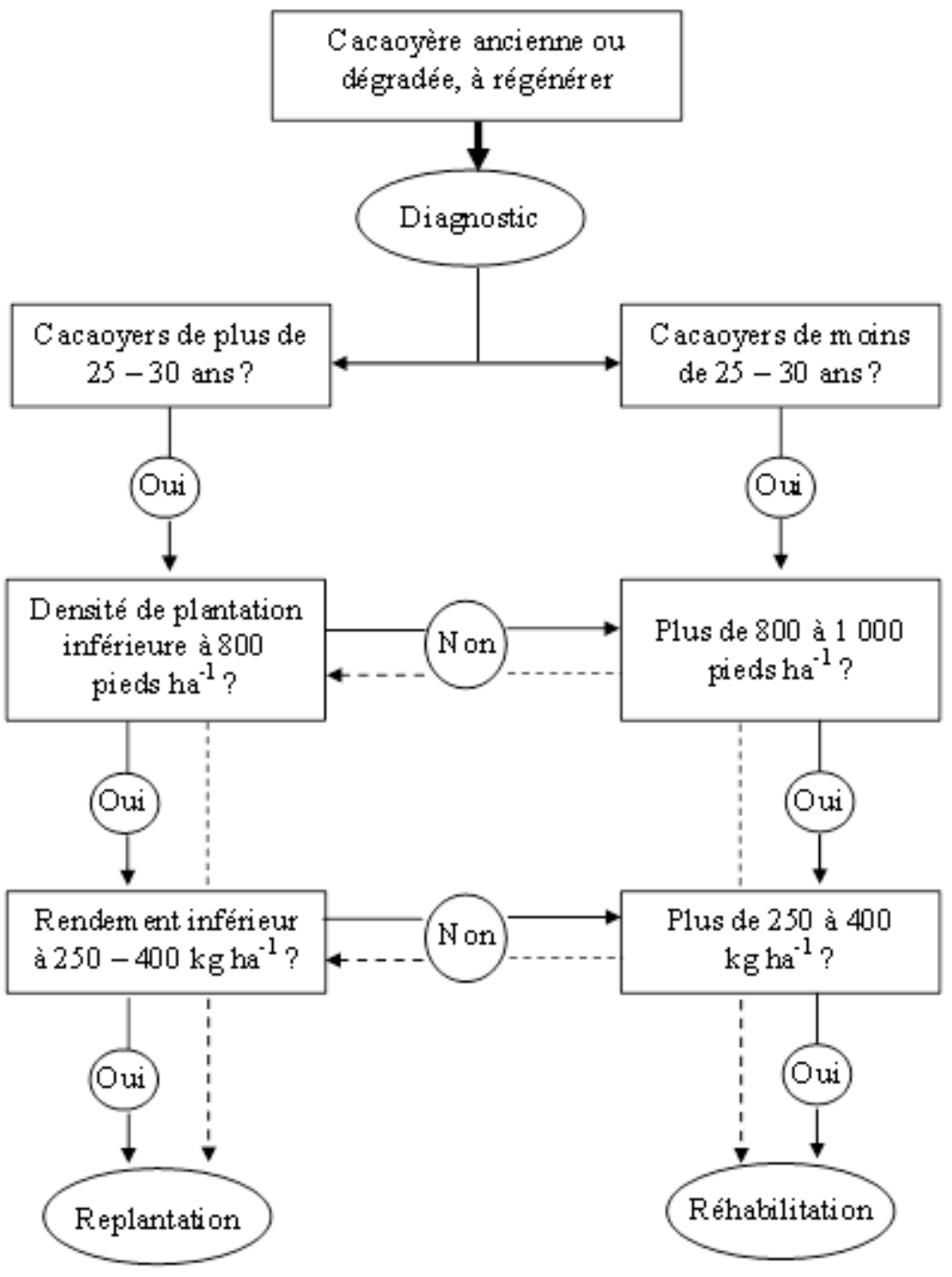

Figure 5. Guide de diagnostic et de décision de l'option de régénération cacaoyère, basé sur l'âge, la densité de plantation et le rendement moyen des vieux vergers de cacaoyers. 
les vergers de cacaoyers avant la réhabilitation ou la replantation étaient significativement différents $(\mathrm{P}<0,0001)$ de par leur âge, la densité des plantations et leurs rendements moyens.

Critères et guide pour le diagnostic des vergers de cacaoyers à régénérer. Les comparaisons faites ont montré qu'en milieu paysan, les critères discriminants des vergers régénérés sont l'âge, la densité de plantation et le rendement. Les moyennes de ces critères pour l'ensemble des plantations enquêtées étaient comprises entre :

(i) 25 et 30 ans, pour l'âge ;

(ii) 800 et 1000 arbres ha $^{-1}$, pour la densité ;

(iii) 250 et $400 \mathrm{~kg} \mathrm{ha}^{-1} \mathrm{an}^{-1}$, pour la production.

Ces critères et ces moyennes ont permis d'élaborer un « guide » (Fig. 5) qui aide à réaliser le diagnostic des vergers de cacaoyers puis à décider de les réhabiliter ou de les replanter.

\section{DISCUSSION}

Les vergers de cacaoyers replantés étaient, dans l'ensemble, d'anciennes plantations dégradées et peu productives. En effet, ils avaient en moyenne 38 ans, avec des densités inférieures à 1000 pieds ha $^{-1}$ et des rendements inférieurs à $200 \mathrm{~kg} \mathrm{ha}^{-1}$. Ces valeurs moyennes sont très faibles, par rapport à plus de 1500 pieds ha ${ }^{-1}$ et environ $400 \mathrm{~kg} \mathrm{ha}^{-1}$ dans les plantations paysannes en Côte d'Ivoire (Freud et al., 2000 ; Assiri et al., 2009). Des vieux vergers de cacaoyers replantés au Togo (Deuss, 1981) et au Ghana (Gyamfi, 1984) avaient des caractéristiques agronomiques similaires. Les résultats obtenus ont montré également que les moyennes d'âge des vergers remplacés diminuaient de l'Est (43 à 46 ans) vers le Sud-Ouest (10 à 26 ans). Le gradient décroissant de l'âge de ces vergers révèle qu'ils sont généralement remplacés plus tardivement à l'Est et au Sud-Est, par rapport aux autres régions. Les différences observées peuvent s'expliquer par la durée économique d'exploitation des cacaoyères qui varie d'une région à l'autre, en fonction des sols et des systèmes de conduite des plantations. En effet, il a été démontré que les sols sont plus favorables à la cacaoculture à l'Est et au Sud-Est que dans la région ouest et sud-ouest du pays. (Ruf, 2000 ; Koko et al., 2009). Par ailleurs, la majorité des plantations y est conduite sous un ombrage permanent (Assiri et al., 2009). Selon Petithuguenin (1995), ces vergers sont plus durables et leurs rendements restent satisfaisants sur une période plus longue, de 20 à 30 ans, quelques fois plus, avant d'être remplacés. Par contre, à l'Ouest et au Sud-Ouest, les cacaoyères se dégradent précocement, après 10 à 15 ans d'exploitation, sous l'effet conjugué de la conduite en plein soleil (Petithuguenin, 1995 ; Ruf, 1999) et des sols gravillonnaires, souvent indurés à moyenne profondeur (Koko, 2008).

En ce qui concerne la réhabilitation, les vergers concernés étaient, dans la majorité des cas $(64,3 \%)$, des cacaoyères de 10 à 30 ans, ayant une densité encore bonne (> 1200 arbres ha ${ }^{-1}$ en moyenne). Il s'agissait, à l'analyse de l'ensemble des autres caractéristiques agronomiques déterminées, de plantations matures et peu dégradées, mais dont les rendements moyens étaient relativement faibles (environ $300 \mathrm{~kg} \mathrm{ha}^{-1}$ ). Ces rendements pouvaient donc être améliorés. En effet, dans cette tranche d'âges (10 à 30 ans), des rendements moyens de $700 \mathrm{~kg} \mathrm{ha}^{-1}$ ont été observées dans certaines plantations paysannes (Assiri et al., 2009) et ont été significativement améliorés à travers la réhabilitation par l'application des bonnes pratiques agricoles et la fertilisation minérale (Assiri et al., 2012).

Ces résultats ont confirmé certains déterminants sociaux et économiques de la replantation cacaoyère décrits par Ruf (2000). Il s'est agi ici du vieillissement ou de la dégradation des vergers, entraînant la chute des rendements et des revenus des producteurs. En effet, dans cette étude, l'état de dégradation (âge et densité de plantation) et le rendement ont constitué les critères majeurs qui ont conduit à la décision de réhabilitation ou de replantation des vergers de cacaoyers.

Dans le cadre de la régénération cacaoyère, l'un des défis majeurs à relever pour assurer une production durable de cacao en Côte d'Ivoire, le guide de diagnostic et de prise de décision proposé au terme de cette étude pourrait constituer un précieux outil de travail pour le conseil agricole. En effet, ils pourraient s'en servir pour caractériser les vergers de cacaoyers en vue 
d'identifier les besoins en matière de réhabilitation et de replantation. Pour les producteurs, le guide pourrait contribuer à une meilleure gestion de leurs vergers de cacaoyers.

\section{REMERCIEMENTS}

Les enquêtes dont certains résultats sont résumés dans le présent article ont été effectuées dans le cadre du projet PIC-Cacao entre le CNRA Côte d'Ivoire et le CIRAD France. Les auteurs renouvellent leurs remerciements aux Ministère français des Affaires Etrangères (MAE) pour le financement de ce projet à travers les Fonds de Solidarité Prioritaire (FSP).

\section{RÉFÉRENCES BIBLIOGRAPHIQUES}

Anonyme. 1998. L'agriculture ivoirienne à l'aube du $21^{\text {ème }}$ siècle. Salon de l'Agriculture et des Ressources Animales d'Abidjan (SARA). Ministère d'État, Ministère de l'Agriculture et des Ressources Animales. Éditions Dialogue Production - Multimédia, Abidjan, Côte d'Ivoire, 295p.

Anonyme. 2003. Filière Café-Cacao : quelles stratégies face aux nouveaux enjeux et défis ? Actes des Journées portes ouvertes 2003 du Café et du Cacao, du 9 au 11 juillet 2003, Abidjan, Côte d'Ivoire. 12p.

Assiri, A.A., Yoro, G.R., Deheuvels, O., Kébé, B.I., Kéli, Z.J., Adiko, A. et Assa, A. 2009. Les caractéristiques agronomiques des vergers de cacaoyer (Theobroma cacao L.) en Côte d'Ivoire. Journal of Animal \& Plant Sciences 2(1): 55-66.

Assiri, A.A. 2010. Etude de la régénération cacaoyère en côte d'ivoire : impact des techniques de réhabilitation et de replantation sur le développement et la productivité des vergers de cacaoyers (Theobroma cacao L.) en relation avec l'état du sol. Thèse de Doctorat Unique de l'UFR STRM, Université de Cocody-Abidjan, Côte d'Ivoire. 170p.

Assiri, A.A., Kacou, E.A., Assi, F.A., Ekra, K.S., Dji, K.F., Couloud, J.Y. et Yapo, A.R. 2012. Rentabilité économique des techniques de réhabilitation et de replantation des vieux vergers de cacaoyers (Theobroma cacao L.) en Côte d'Ivoire. Journal of Animal \& Plant Sciences 14 (2): 1939-1951.

Bardin, A. 1937. Le cacaoyer en Côte d'Ivoire. Annales Agricoles de l'Afrique occidentale française et étrangère 1 (2) : 135-150.

Brou, Y.T. 2005. Climat, mutations socioéconomiques et paysages en Côte d'Ivoire. Mémoire de synthèse des activités scientifiques présenté en vue de l'obtention de l'Habilitation à Diriger des Recherches, Université des Sciences et Technologies de Lille, France. 212p.

Burle, L. 1962. Le cacaoyer. Tome deuxième. Éditions G.-P. Maisonneuve et Larose, Paris, France : pp. 486-491.

Deuss, J. 1981. La vulgarisation cacaoyère au Togo. Café Cacao Thé 25 (1) : 37-44.

FAO. 2003. L'étude prospective du secteur forestier en Afrique (FOSA) : Document national de prospective - Côte d'Ivoire. http:/ /www.fao.org/docrep/003/X6780F/ X6780F00.htm. Accédé le 11 novembre 2008.

FAO. 2009. Harmonized world soil database (HWSD). FAO (Food and Agriculture Organization), Rome, Italy. 38p.

Freud, E.H., Petithuguenin, P. et Richard, J. 2000. Les champs de cacao : un défi de compétitivité Afrique-Asie. Éditions Karthala et CIRAD, Collection Économie et développement, Paris, France. 207p.

Gyamfi, A.A. 1984. Rehabilitation of the cocoa industry (farm development programmes). Proceedings of the $9^{\text {th }}$ international cocoa research conference, February 12-18, 1984, Lomé, Togo: 635-640.

ICCO. 2015. Quarterly Bulletin of Cocoa Statistics, Vol. XLI, No. 4, Cocoa year 2014/15. International Cocoa Organization (ICCO), Westgate House, London, United Kingdom. http://www.icco.org/about-us/internationalcocoa-agreements/cat view/30-relateddocuments/46-statistics-production.html, Accessed 19 février 2016.

Jouve, P. et Milly, H. 1990. Compétitivité du cacao africain. Rapport d'étude. Ministère de la coopération et du développement, Paris, France. Rapport d'étude, 279 p.

Kassin, K.E., Doffangui, K., Yoro, G.R. et Assa, A. 2008. Variabilité pluviométrique et 
perspectives pour la replantation cacaoyère dans le Centre Ouest de la Côte d'Ivoire. Journal of Applied Biosciences 12 : 633 - 641.

Koko, L.K. 2008. Influence des caractères morphopédologiques et chimiques des sols sur la dégradation précoce des cacaoyers dans le Sud-Ouest de la Côte d'Ivoire : Cas de Méagui et de San-Pedro. Thèse de Doctorat unique, UFR STRM, Université de Cocody, Abidjan, Côte d'Ivoire, $120 \mathrm{p}$.

Koko, L.K., Kassin, K.E., Yoro, G., Ngoran, K., Assiri, A.A. et Yao-Kouamé, A. 2009. Corrélations entre le vieillissement précoce des cacaoyers et les caractéristiques morphopédologiques dans le Sud-Ouest de la Côte d'Ivoire. Journal of Applied Biosciences 24: 1508-1519.

Losch, B. 1994. Cacaos africains : Comment la Côte d'Ivoire a devancé le Cameroun. Plantations, Recherche, Développement 1 (2) : 15-27.

Naï Naï, S., Cheyns, E. et Ruf, F. 2000. Adoption du palmier à huile en Côte d'Ivoire. Oléagineux, Corps Gras, Lipides 7 (2) : 155165.

Perraud, A. 1971. Les sols. Le milieu naturel de la Côte d'Ivoire. Mémoire ORSTOM 50 : 69-390.

Petithuguenin, P. 1995. Cacaoculture et évolution du milieu : Une contribution à la réflexion sur la reproductibilité de ces systèmes de cultures. Actes du séminaire sur la fertilité du milieu et stratégies paysannes sous les tropiques humides, du 13 au 17 Novembre 1995, Montpellier, France : pp. 340-349.

Petithuguenin, P. 1998. Les conditions naturelles de production du cacao en Côte d'Ivoire, au Ghana et en Indonésie. Plantations, recherche, développement, NovembreDécembre 1998 : pp. 393 - 411.

Ruf, F. 1995. Booms et crises du cacao : les vertiges de l'or brun. Éditions Karthala \& CIRAD, Collection Économie et développement, Paris, France. $459 \mathrm{p}$.

Ruf, F. 1999. Comment et pourquoi la Côte d'Ivoire produit durablement plus d'un million de tonnes de cacao. Afrique Agriculture 268 : 21-25.

Ruf, F. 2000. Déterminants sociaux et économiques de la replantation. Oléagineux, Corps Gras, Lipides 7 (2) : 189-196.

Ruf, F. et Allangba, K. 2001. Les difficultés de la replantation. Quel avenir pour le cacao en Côte d'Ivoire. Actes de la conférence internationale sur l'avenir des cultures pérennes: Investissement et durabilité en zones tropicales humides, 5 au 9 Juin 2001, Yamoussoukro (Côte d'Ivoire). CD-ROM du CIRAD-DIST, $13 \mathrm{p}$. 